Article

\title{
A Dynamic State-of-Charge Estimation Method for Electric Vehicle Lithium-Ion Batteries
}

\author{
Xintian Liu ${ }^{1}$, Xuhui Deng ${ }^{1}$, Yao He ${ }^{1}{ }^{1}$, Xinxin Zheng ${ }^{1}$ and Guojian Zeng ${ }^{2}$ \\ 1 Automobile Engineering Technology Research Institution, Hefei University of Technology, Hefei 230009, \\ China; xintian.liu@hfut.edu.cn (X.L.); xuhdeng@163.com (X.D.); xinxin.zheng@hfut.edu.cn (X.Z.) \\ 2 Anhui Ruineng Technology Company, Hefei 230011, China; guojian.zeng@rntec.net \\ * Correspondence: yao.he@hfut.edu.cn
}

Received: 26 November 2019; Accepted: 17 December 2019; Published: 25 December 2019

\begin{abstract}
With the increasing environmental concerns, plug-in electric vehicles will eventually become the main transportation tools in future smart cities. As a key component and the main power source, lithium-ion batteries have been an important object of research studies. In order to efficiently control electric vehicle powertrains, the state of charge (SOC) of lithium-ion batteries must be accurately estimated by the battery management system. This paper aims to provide a more accurate dynamic SOC estimation method for lithium-ion batteries. A dynamic Thevenin model with variable parameters affected by the temperature and SOC is established to model the battery. An unscented Kalman particle filter (UPF) algorithm is proposed based on the unscented Kalman filter (UKF) algorithm and the particle filter (PF) algorithm to generate nonlinear particle filter according to the advantages and disadvantages of various commonly used filtering algorithms. The simulation results show that the unscented Kalman particle filter algorithm based on the dynamic Thevenin model can predict the $\mathrm{SOC}$ in real time and it also has strong robustness against noises.
\end{abstract}

Keywords: lithium-ion battery; dynamic thevnin model; state of charge; unscented Kalman particle filter

\section{Introduction}

With the development of renewable energy and the wide concern on global warming, plug-in electric vehicles become the main transportation tool in reducing transport emissions for future smart cities. For plug-in electric vehicles, the energy is mainly provided by lithium-ion batteries, which are charged through an electric power supply network that is preferably powered by clean renewable energy sources. In order to properly control the electric vehicles to meet acceleration, braking, and normal driving needs, the battery management system must properly control the charging/discharging status of the lithium-ion battery pack, and such control is usually based on accurate monitoring and estimation of key battery parameters such as the battery state of charge (SOC). SOC reflects the remaining battery capacity directly [1], which can be translated to the remaining mileage that the vehicle can travel [2]. Therefore, accurate estimation of SOC is an important requirement to manage battery power [3], predict remaining mileage [4], and avoid battery overcharging or over discharging [5].

In recent years, various methods have been proposed to estimate the SOC, including ampere-hour integral method [6], open-circuit voltage method [7], neural network method [8], and model-based estimating method. Open-circuit voltage method is simple in principle, but it cannot achieve online estimation of SOC [7]. The ampere-hour integral method is a common open-loop SOC estimation method [6], but this method requires the initial SOC value, and the error generated in the current measurement process is inevitable. In the actual SOC estimation process, its cumulative error is always increasing, which cannot satisfy the real-time and accurate SOC estimation requirement. Neural 
network method does not need an accurate model [8], but it needs a large number of data training samples in the actual estimation. Different training samples and methods will affect the accuracy of estimation.

Compared with the non-model-based method mentioned above, model-based methods are widely used due to their characteristics of easily identifiable parameters and robustness. Popular battery models include the electrochemical model [9], the equivalent circuit model [10], and the impedance spectrum model [11]. Because of the high nonlinearity in the actual working process of batteries, this method often combines nonlinear system estimation algorithms to complete the estimation of battery SOC. Among all these estimation methods, the extended Kalman filter (EKF) algorithm is very popular [12]. This algorithm needs to linearize the nonlinear system in the actual estimation process, and it has the disadvantage of performance degradation or divergence when solving nonlinear problems. To deal with this problem, the unscented Kalman filter (UKF) algorithm [13] is proposed. It generates the sample points through unscented transformation. This can not only avoid linearizing the nonlinear equation, but also reflect more characteristics of the system, which is helpful to improve the estimation accuracy. As an effective algorithm to solve nonlinear problems, the particle filter algorithm can be applied to the highly nonlinear characteristics of a power battery system [14]. It uses sampling approximation with different probability distributions to overcome the disadvantage of the Kalman filtering algorithm, which can only be used for the Gaussian noise density distribution of a linear system. However, due to the lack of inherent particles and the high computation complexity, the system estimation accuracy is adversely affected. This paper aims to propose a new SOC estimation method combining the advantages of the above algorithms, that is, the particle filter algorithm will be applied to generate the proposed distribution of nonlinear particle filter, and it is further combined with the unscented Kalman particle filter (UPF) algorithm to estimate SOC.

Note that the accuracy of SOC estimation is affected by not only the accuracy of the filtering algorithm, but also the accuracy of the battery model. The battery model describes the relationship among voltage, current, temperature, SOC, and other factors. When selecting the battery model, the accuracy and complexity of the battery model should be considered. Thevenin model has been widely used in battery modeling due to its advantages such as simple physical structure and easy experiment identification for model parameters [15]. However, its parameters are greatly affected by other factors, such as temperature and SOC [16]. In this paper, Thevenin model with dynamic parameters varying against temperature and SOC is adopted to characterize the battery.

Different from [9], the two-level factor experimental design method [17] and least squares [18] method are applied together with the aforementioned Thevenin Battery model to conduct piecewise fitting of model parameters, and an improved battery model with variable parameters will be obtained finally. Then, the advantages of UPF algorithm are analyzed based on the obtained battery Thevenin model, and the SOC state estimation process based on the UPF algorithm is analyzed. Simulation results show that the UPF algorithm based on the dynamic battery model can accurately reflect the SOC of a battery in real time and has a strong robustness against noise.

This paper is organized as follows. Section 2 presents the battery Thevenin model, and the corresponding model parameters are identified in Section 3. Section 4 introduces the new SOC estimation method, which is further validated by the simulation results in Section 5. Conclusions are drawn in Section 6.

\section{Battery Thevenin Model}

According to the principle of Thevenin equivalence, a Thevenin model whose parameters are affected by temperature $T$ and SOC is established, as shown in Figure 1 [19]:

According to Figure 1, the open-circuit voltage $U_{o c}$ can be calculated as follows:

$$
\left\{\begin{array}{l}
U_{o c}=U_{1}+U_{p o l}+\left(R_{i}+R_{o h m}\right) I_{1} \\
\dot{U}_{p o l}=\frac{I_{1}}{C_{p o l}}-\frac{U_{p o l}}{C_{p o l} R_{p o l}}
\end{array}\right.
$$


where $U_{1}$ represents terminal voltage, $R_{p o l}$ and $C_{p o l}$ represent the polarization resistance and the polarization capacitance, respectively; the terminal voltage of $C_{p o l}$ is the polarization voltage $U_{p o l} . R_{\text {ohm }}$ and $R_{i}$ are the ohmic internal resistance and the other internal resistance, respectively, and $I_{1}$ is the battery charging or discharging current. Note that $C_{\text {pol }}, R_{\text {pol }}, R_{\text {ohm }}$, and $R_{i}$ are all variable parameters affected by battery SOC and temperature $T$. A single lithium-ion battery with a rated capacity of $9 \mathrm{Ah}$ is selected, and the parameters are measured by the hybrid impulse characteristics experiment (HPPC) [20] in order to better fit the model parameters.

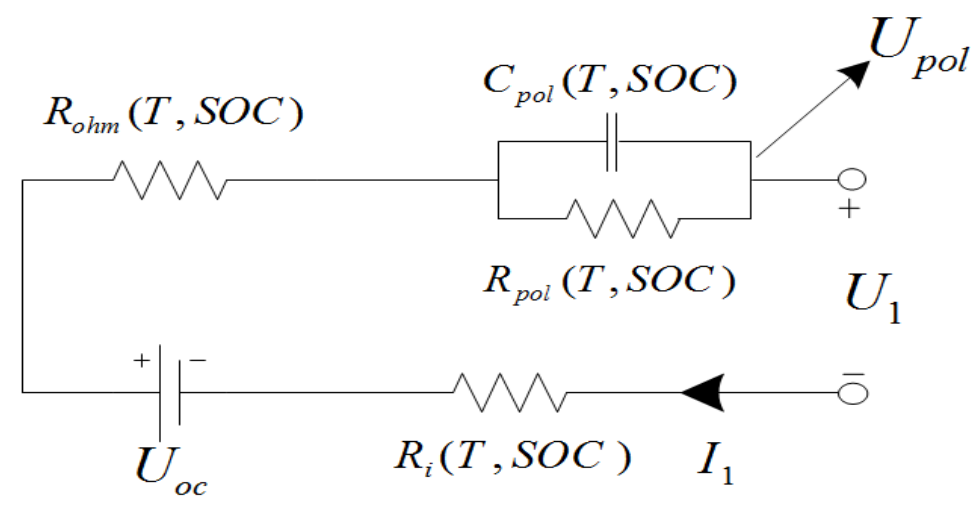

Figure 1. Variable parameter Thevenin model. SOC, state of charge.

Figure 2 shows the relationship among $R_{\text {ohm }}$, SOC, and T measured by multiple HPPC experiments. $R_{\text {ohm }}$ changes simultaneously with both $T$ and SOC when SOC is less than 0.3 . This $R_{\text {ohm }}$ changes mainly with $T$ when SOC is greater than 0.3 , and it is slightly higher when SOC is less than 0.7 . Therefore, the $R_{\text {ohm }}$ can be characterized when SOC is considered separately over the three different intervals, [0, 0.3], $(0.3,0.7]$, and $(0.7,1]$.

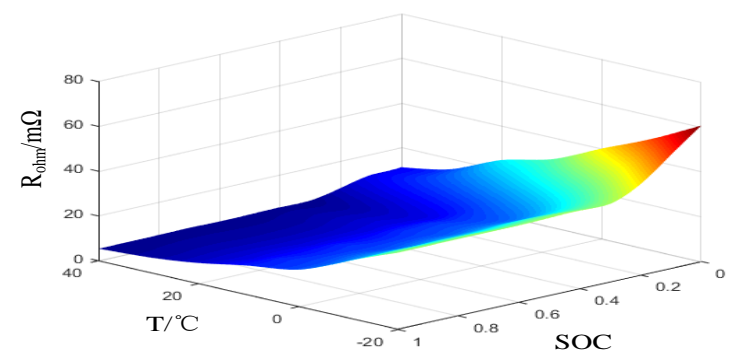

Figure 2. Relationship among the measured $R_{o h m}, \mathrm{SOC}$, and $T$.

Similar to the above analysis, the relationship among $R_{\text {pol }}$, SOC, and $T$ can be measured by HPPC experiments, as shown in Figure 3. We can see that $R_{\text {pol }}$ in Figure 3 has similar variation patterns to those seen in Figure 2, thus $R_{p o l}$ can also be analyzed by dividing SOC into $[0,0.3],(0.3,0.7]$, and $(0.7,1]$.

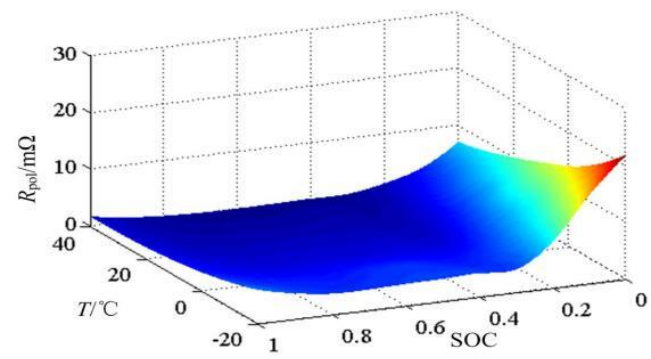

Figure 3. Relationship among the measured $R_{p o l}, \mathrm{SOC}$, and $T$. 


\section{Identification of Battery Model Parameters}

In order to obtain the actual relationship between $R_{o h m}, R_{p o l}, \mathrm{SOC}$, and $T$, a discrete data-fitting method can be used to fit the measured resistance values at different temperature points under different SOCs, and the fitting method is as follows.

\subsection{Identification of Parameters $R_{\text {ohm }}$ and $R_{\text {pol }}$}

The above analysis shows that $R_{o h m}$ and $R_{p o l}$ change with $T$ and SOC simultaneously when the SOC is no more than 0.3 . In order to establish a more accurate battery model while still having a simple battery model, the SOC and $T$ can be used as input for the equivalent internal resistance model by the two-level factorial design of experimental (DOE) fitting method. Central composite design (CCD) is the most common DOE method because of its simple design and strong system correlations. In this paper, the DOE based on CCD is applied to the internal resistance model, and the internal resistance is fitted by using $T$ and $S O C$ as inputs. The fitted equivalent internal resistance model includes the first-order, second-order, and cross terms, and the fitting principle is shown in Figure 4.

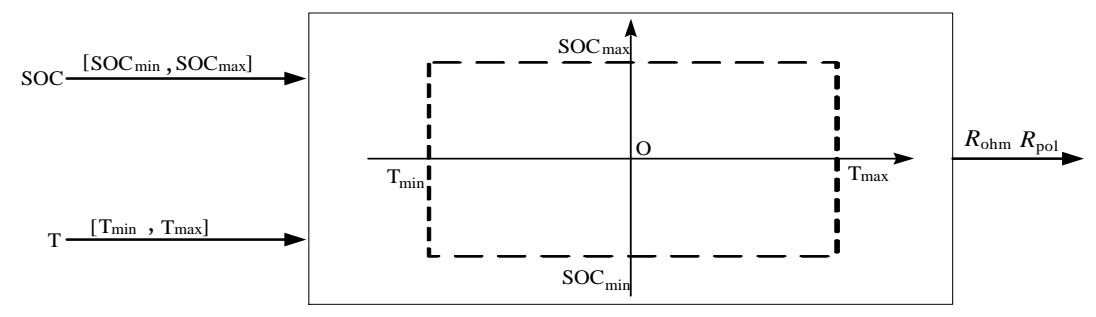

Figure 4. Internal resistance DOE (design of experimental) model based on CCD (central composite design).

The temperature $T$ is the main factor that affects the internal resistance change, and the internal resistance can be fitted by the least squares method using the temperature $T$ as the input when the $\mathrm{SOC}$ is greater than 0.3 .

Through the above analysis, $R_{o h m}$ and $R_{p o l}$ can be fitted as shown in Equation (2). The corresponding piecewise fitted equation is as follows:

$$
R_{\text {ohm }}(\mathrm{SOC}, T)= \begin{cases}a_{0} T+a_{1} \mathrm{SOC}+a_{2} \mathrm{SOCT}+a_{3} T^{2}+a_{4} \mathrm{SOC}^{2}+a_{5} & 0 \leq \mathrm{SOC} \leq 0.3 \\ a_{6} e^{-a_{7} T}+a_{8} & 0.3<\mathrm{SOC} \leq 0.7 \\ a_{9} e^{-a_{10} T}+a_{11} & 0.7<\mathrm{SOC} \leq 1\end{cases}
$$

Each parameter in Table 1 is the average of the same type and the same batch of multiple battery cells for many times. In practical applications, there should be a confidence interval of $95 \%$ confidence level because there will be a large number of batteries, and the same kind of battery cell parameters are basically different, then the piecewise fitted relationship diagram of $R_{\text {ohm }}$ against SOC and Tis shown in Figure 5.

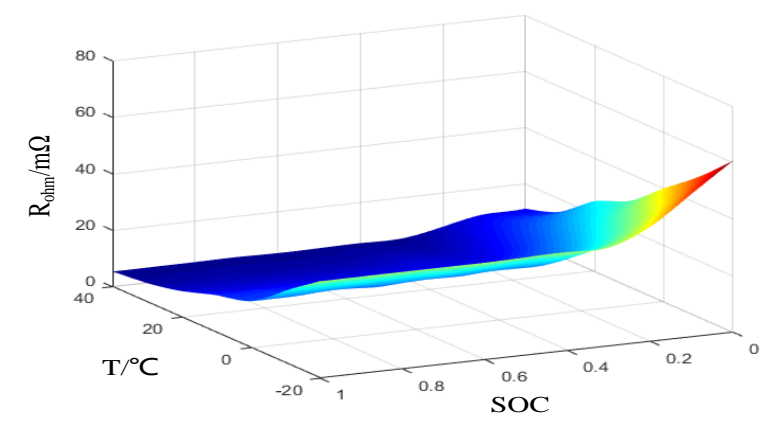

Figure 5. Relationship among fitted $R_{o h m}$ and SOC, T. 
Table 1. Parameters of $R_{\text {ohm }}$.

\begin{tabular}{ccc}
\hline Parameter & Average & Confidence Interval (95\% Confidence Level) \\
\hline$a_{0}$ & -0.8908 & $(-0.9353,-0.8462)$ \\
$a_{1}$ & -107.899 & $(-113.2939,-102.504)$ \\
$a_{2}$ & 0.8831 & $(0.8389,0.9272)$ \\
$a_{3}$ & 0.007642 & $(0.00726,0.008024)$ \\
$a_{4}$ & 111.2554 & $(105.6926,116.8182)$ \\
$a_{5}$ & 40.4614 & $(38.4383,42.4845)$ \\
$a_{6}$ & 11.1194 & $(10.5634,11.6754)$ \\
$a_{7}$ & 0.0273 & $(0.02474,0.02986)$ \\
$a_{8}$ & 2.371 & $(2.2234,2.5186)$ \\
$a_{9}$ & 10.8226 & $(10.2618,11.3834)$ \\
$a_{10}$ & 0.0293 & $(0.02763,0.03097)$ \\
$a_{11}$ & 2.182 & $(2.051,2.313)$ \\
\hline
\end{tabular}

Following a similar analysis as above, the piecewise fitted equation of $R_{p o l}$ and the relation between $R_{p o l}$ and SOC, $T$ can be obtained as follows:

$$
R_{p o l}(\mathrm{SOC}, T)= \begin{cases}b_{0} T+b_{1} \mathrm{SOC}+b_{2} \mathrm{SOC} T+b_{3} T^{2}+b_{4} \mathrm{SOC}^{2}+b_{5} & 0 \leq \mathrm{SOC} \leq 0.3 \\ b_{6} e^{-b_{7} T}+b_{8} & 0.3<\mathrm{SOC} \leq 0.7 \\ b_{9} e^{-b_{10} T}+b_{11} & 0.7<\mathrm{SOC} \leq 1\end{cases}
$$

In practical application, the parameters in Table 2 should also have confidence intervals of $95 \%$ confidence level, and the relationship between $R_{p o l}$ and SOC, T obtained by piecewise fitting is given as Figure 6.

Table 2. Parameters of $R_{p o l}$.

\begin{tabular}{ccc}
\hline Parameter & Average & Confidence Interval (95\% Confidence Level) \\
\hline$b_{0}$ & -0.431 & $(-0.4582,-0.4038)$ \\
$b_{1}$ & -58.3 & $(-61.77,-54.83)$ \\
$b_{2}$ & 0.5375 & $(0.4804,0.5946)$ \\
$b_{3}$ & 0.00214 & $(0.001863,0.002417)$ \\
$b_{4}$ & 86.15 & $(81.51,90.79)$ \\
$b_{5}$ & 16.82 & $(15.835,17.805)$ \\
$b_{6}$ & 1.5988 & $(1.459,1.7386)$ \\
$b_{7}$ & 0.02103 & $(0.01329,0.02877)$ \\
$b_{8}$ & -0.7334 & $(-0.7736,-0.6932)$ \\
$b_{9}$ & 1.952 & $(1.586,2.318)$ \\
$b_{10}$ & 0.01961 & $(0.01457,0.02465)$ \\
$b_{11}$ & -0.7613 & $(-0.8119,-0.7107)$ \\
\hline
\end{tabular}

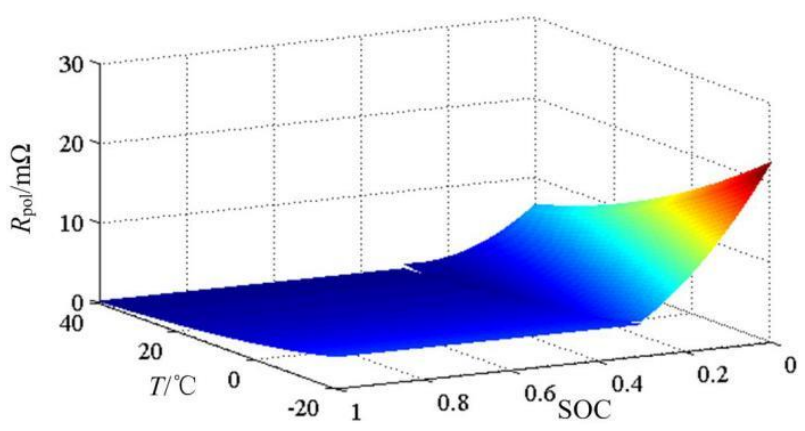

Figure 6. Relationship among fitted $R_{p o l}$ and SOC, $T$. 
$R_{o h m}$ and $R_{p o l}$ are fitted by SOC and T in Figures 5 and 6 following the values measured by the HPPC test in Figures 2 and 3, respectively. Through correlation curve analysis, it is also possible to verify that $R_{\text {ohm }}$ and $R_{\text {pol }}$ will change with the temperature $T$ and battery SOC when SOC is no more than 0.3 ; and change mainly with temperature $T$ when SOC is greater than 0.3 . In other words, the fitted $R_{o h m}$ and $R_{p o l}$ have higher accuracy. Compared with $R_{o h m}$ and $R_{p o l}$, the other internal resistance $R_{i}$ can be ignored in the battery model since it is relatively much smaller.

\subsection{Identification of Parameter $C_{\text {pol }}$}

Parameter $\tau$ can be identified by the charge-discharge experiments, because the polarization voltage will decay $95 \%$ after $3 \tau$ time when the battery stops discharging. Table 3 gives the measured values of $\tau$ at different typical temperatures.

Table 3. Measurement of $\tau$ at typical temperature.

\begin{tabular}{ccccccc}
\hline Temperature $\left({ }^{\circ} \mathbf{C}\right)$ & $\mathbf{- 1 0}$ & $\mathbf{0}$ & $\mathbf{1 0}$ & $\mathbf{2 0}$ & $\mathbf{3 0}$ & $\mathbf{4 0}$ \\
\hline$\tau(\mathrm{s})$ & 14.1 & 11.4 & 9.3 & 7.8 & 6.7 & 5.9 \\
\hline
\end{tabular}

The fitted equation of the parameter $\tau$ is obtained through the analysis of the measurement data, where the parameters in the equation are identified in Table 4 , and the parameters in Table 4 should have a confidence level of $95 \%$ confidence level in practical application.

$$
\tau=c_{0} e^{-c_{1} T}+c_{2}
$$

Table 4. Parameters of $\tau$.

\begin{tabular}{ccc}
\hline Parameter & Average & Confidence Interval (95\% Confidence Level) \\
\hline$c_{0}$ & 7.784 & $(7.358,8.211)$ \\
$c_{1}$ & 0.03034 & $(0.02817,0.0325)$ \\
$c_{2}$ & 3.573 & $(3.183,3.962)$ \\
\hline
\end{tabular}

The equation of $C_{\text {pol }}$ is given as follows:

$$
C_{p o l}=\frac{\tau}{R_{p o l}(\mathrm{SOC}, T)}
$$

\section{SOC Estimation}

To characterize the influence of temperature on SOC estimation, the SOC state equation can be expressed as follows:

$$
\operatorname{SOC}(t)=\operatorname{SOC}(t-1)-\int_{t-1}^{t} \frac{\eta i(\tau) d \tau}{\lambda Q_{f u l l}}
$$

where $\operatorname{SOC}(t)$ and $\operatorname{SOC}(t-1)$ in the formula represent SOC values of time $t$ and time $t-1$ respectively; $\lambda$ represents a coefficient which changes under different temperatures(see Figure 7$) ; i(\tau)$ is the charging-discharging current; $T$ denotes temperature; and $Q_{\text {full }}$ represents the nominal capacity of battery at normal temperature. In order to establish a more accurate battery model while keeping a simple model structure, the charge discharge efficiency is taken as 1 [21].

From Figure $7, \lambda$ can be identified through data fitting as follows:

$$
\lambda=l_{0} e^{-\frac{T}{l_{1}}}+l_{2} e^{-\frac{T}{l_{3}}}+l_{4}
$$

Its parameter values are shown in Table 5. 


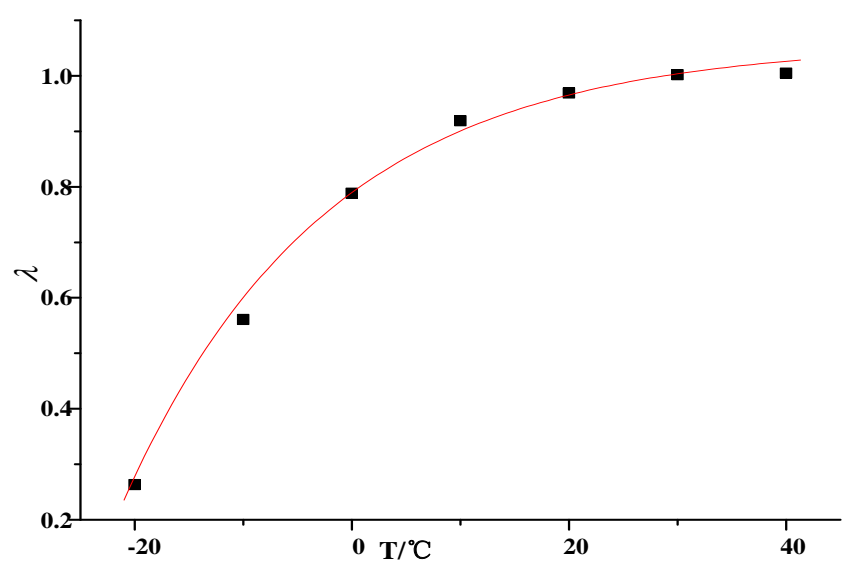

Figure 7. The value $\lambda$ of at different temperature.

Table 5. Parameter identification of $\lambda$.

\begin{tabular}{ccc}
\hline Parameter & Average & Confidence Interval (95\% Confidence Level) \\
\hline$l_{0}$ & -0.13284 & $(-0.34114,0.07546)$ \\
$l_{1}$ & 18.2064 & $(14.5549,21.8579)$ \\
$l_{2}$ & -0.13264 & $(-0.34094,0.07566)$ \\
$l_{3}$ & 18.2186 & $(14.5521,21.8851)$ \\
$l_{4}$ & 1.04882 & $(-1.01516,3.1128)$ \\
\hline
\end{tabular}

According to the variable parameter Thevenin model and Equation (1), the following equation is obtained:

$$
U_{1}(\mathrm{SOC}, T)=U_{o c}-U_{p o l}(\mathrm{SOC}, T)-I_{1} R_{o h m}(\mathrm{SOC}, T)
$$

The discrete state-space equations can be obtained by discretizing Equations (6) and (8) as follows:

$$
\begin{gathered}
x_{k+1}=f\left(x_{k}, u_{k}\right)+w_{k}=x_{k}-\frac{\eta u_{k} \Delta t}{\lambda Q_{f u l l}}+w_{k} \\
y_{k}=g\left(x_{k}, u_{k}\right)+v_{k}=U_{o c}-U_{p o l}\left(x_{k}, T\right)-u_{k} R_{o h m}\left(x_{k}, T\right)+v_{k}
\end{gathered}
$$

where $x_{k}$ represents the $k$ th calculated SOC, $\Delta t$ represents the sampling period; $w_{k}$ and $v_{k}$ represent the system state noise and measurement noise, respectively; $w_{k}$ and $v_{k}$ both have zero-means and their covariances are the uncorrelated Gaussian white noise of $Q$ and $R$, respectively. The observation variable $y_{k}$ characterizes the terminal voltage $U_{1}$ calculated by the kth calculation of the battery model, and the input variable $u_{k}$ characterizes the battery charge-discharge current $I_{1}$ during the $k$ th calculation.

According to the state space equation, the process for achieving the state of lithium battery SOC estimation by unscented Kalman particle filter algorithm is given as follows:

(1) Using $(0,1)$ uniform distribution to generate $N$ initial values of SOC, the $N$ initial state particles and covariance are obtained as follows:

$$
\begin{gathered}
X_{0}^{(i)+}=\left[\begin{array}{lll}
x_{0}^{(i)+} & 0 & 0
\end{array}\right]^{T} \\
x_{0}^{(i)+}=\operatorname{SOC}_{0}^{(i)+} \\
q_{i}=\frac{1}{N}
\end{gathered}
$$




$$
P_{0}^{(i)+}=\left[\begin{array}{ccc}
P_{0} & 0 & 0 \\
0 & Q_{0} & 0 \\
0 & 0 & R_{0}
\end{array}\right]
$$

$P_{0}$ is the initial value of the covariance of each particle. $Q_{0}$ and $R_{0}$ are initial values of system and observation noises.

(2) The proposed state estimation principle is shown in Figure 8, and is further explained below in steps (2.1) and (2.2).

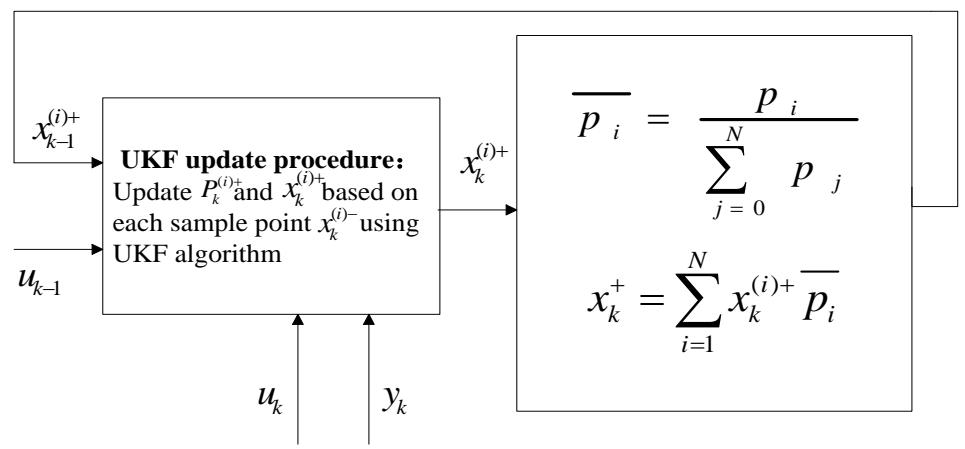

Figure 8. Principle of UPF (unscented Kalman particle filter) state estimation.

The state variables are as follows:

$$
\begin{gathered}
X_{k-1}^{(i)+}=\left[\begin{array}{ccc}
x_{k-1}^{(i)+} & W_{k-1}^{(i)+} & V_{k-1}^{(i)+}
\end{array}\right]^{T} \\
x_{k-1}^{(i)+}=\mathrm{SOC}_{k-1}^{(i)+}
\end{gathered}
$$

The variance of state is as follows:

$$
\bar{P}_{k-1}^{(i)+}=\left[\begin{array}{ccc}
P_{k-1}^{(i)+} & 0 & 0 \\
0 & Q_{k-1}^{(i)+} & 0 \\
0 & 0 & R_{k-1}^{(i)+}
\end{array}\right]
$$

(2.1) The specific estimation process of UPF algorithm is given as follows:

An unscented Kalman filter algorithm is used for each sample point to calculate $x_{k}^{(i)+}$ and $P_{k}^{(i)+}$. The transmission and estimation of system noise and observation noise are considered in this algorithm. The specific steps are given as follows:

(2.1.1) Particle Sigma Point Sampling:

$$
\bar{X}_{k-1}^{(i)+}=\left[\begin{array}{lll}
X_{k-1}^{(i)+} & X_{k-1}^{(i)+}+\sqrt{(n+\lambda) \bar{P}_{k-1}^{(i)+}} & X_{k-1}^{(i)+}-\sqrt{(n+\lambda) \bar{P}_{k-1}^{(i)+}}
\end{array}\right]
$$

At this point, the sampling point set becomes $\left\{\bar{X}_{j, k-1}^{(i)+}, j=1,2 \ldots .2 M, M=n+q+r\right\}$, where $q$ and $r$ are the dimensions of $Q$ and $R$, respectively. $\bar{X}_{j, x, k-1}^{(i)+}, \bar{X}_{j, y, k-1}^{(i)+}, \bar{X}_{j, z, k-1}^{(i)+}$ are the first $\mathrm{n}$ dimensional column vector components, $n+1$ to $n+q$ dimensional column vector components, and $n+q+1$ to $n+$ $r$ dimensional column vector components of the sample point respectively.

(2.1.2) Particle and estimation error covariance time update process:

$$
\bar{X}_{j, x, k}^{(i)-}=f\left(\bar{X}_{j, x, k-1}^{(i)+}, u_{k-1}, \bar{X}_{j, y, k-1}^{(i)+}\right)
$$




$$
\begin{gathered}
x_{k}^{(i)-}=\sum_{j=0}^{2 M} W_{m}^{j} \bar{X}_{j, x, k}^{(i)-} \\
Z_{j, k}^{(i)-}=g\left(\bar{X}_{j, x, k}^{(i)-} u_{k}, \bar{X}_{j, z, k-1}^{(i)-}\right) \\
y_{k}^{(i)-}=\sum_{j=0}^{2 M} W_{m}^{j} Z_{j, k}^{(i)-} \\
P_{k}^{(i)-}=\sum_{j=0}^{2 M} W_{e}^{j}\left(\bar{X}_{j, x, k}^{(i)-}-x_{k}^{(i)-}\right)\left(\bar{X}_{j, x, k}^{(i)-}-x_{k}^{(i)-}\right)^{T}
\end{gathered}
$$

(2.1.3) Particle measurement update process:

$$
\begin{gathered}
P_{x_{k}, y_{k}}^{(i)}=\sum_{j=0}^{2 M} W_{c}^{j}\left(\bar{X}_{j, x, k}^{(i)-}-x_{k}^{(i)-}\right)\left(Z_{j, k}^{(i)-}-y_{k}^{(i)-}\right)^{T} \\
P_{y_{k}, y_{k}}^{(i)}=\sum_{j=0}^{2 M} W_{c}^{j}\left(Z_{j, k}^{(i)-}-y_{k}^{(i)-}\right)\left(Z_{j, k}^{(i)-}-y_{k}^{(i)-}\right)^{T} \\
L_{k}=P_{x_{k}, y_{k}}^{(i)}\left[P_{y_{k}, y_{k}}^{(i)}\right]^{-1} \\
x_{k}^{(i)+}=x_{k}^{(i)-}+L_{k}\left(y_{k}-y_{k}^{(i)-}\right) \\
P_{k}^{(i)+}=P_{k}^{(i)-}-L_{k} P_{y_{k}, y_{k}}^{(i)} L_{k}^{T}
\end{gathered}
$$

(2.2) Resampling phase

The posterior probability $p_{i}$ of particle $x_{k}^{(i)+}$ is calculated from the measurement result $y_{k}$ :

$$
p_{i}=p\left(y_{k} \mid x_{k}^{(i)+}\right)=\frac{1}{\sqrt{2 \pi R}} e^{\frac{-\left[y_{k}-g\left(x_{k}^{(i)+}, i_{k-1}\right)-v_{k}\right]^{2}}{2 R}}
$$

This can be normalized as follows.

$$
\bar{p}_{i}=\frac{p_{i}}{\sum_{j=1}^{N} p_{j}}
$$

Thus, a set of particles $x_{k}^{(i)+}$, error covariance $P_{k}^{(i)+}$, and corresponding probability $\bar{p}_{i}, i=1,2, \ldots, N$ are obtained.

(2.3) Update SOC value:

$$
x_{k}^{+}=\sum_{i=1}^{N} x_{k}^{(i)+} \bar{p}_{i}
$$

\section{Simulation and Validation}

\subsection{Battery Model Validation}

A single $\mathrm{LiFePO}_{4}$ battery with rated capacity of $9 \mathrm{Ah}$ was selected to conduct charge-discharge experiments at different temperatures, and the experimental test platform physical map under different conditions is shown in Figure 9. 


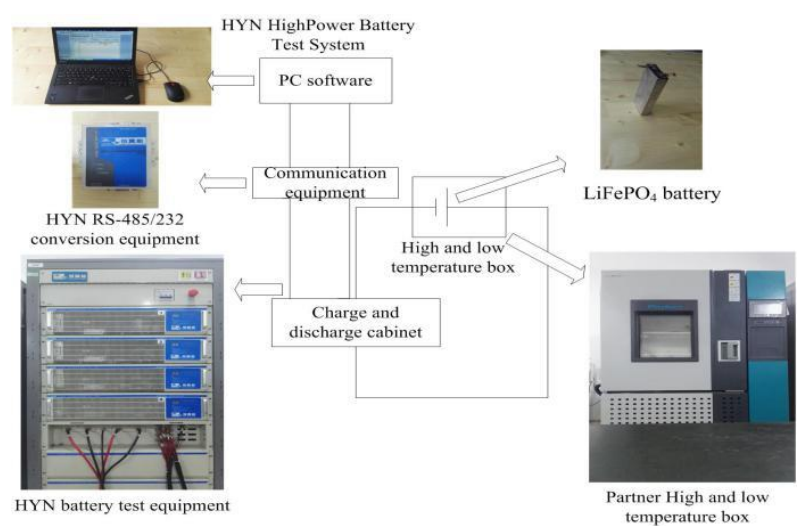

Figure 9. Physical map of experimental test platform.

This paper selected 20 cycles of dynamic stress test (DST) for verification. Figure 10a shows the DST current curve. Taking the temperature $25^{\circ} \mathrm{C}$ as an example, the measurement voltage and the simulation are shown in Figure 10b.

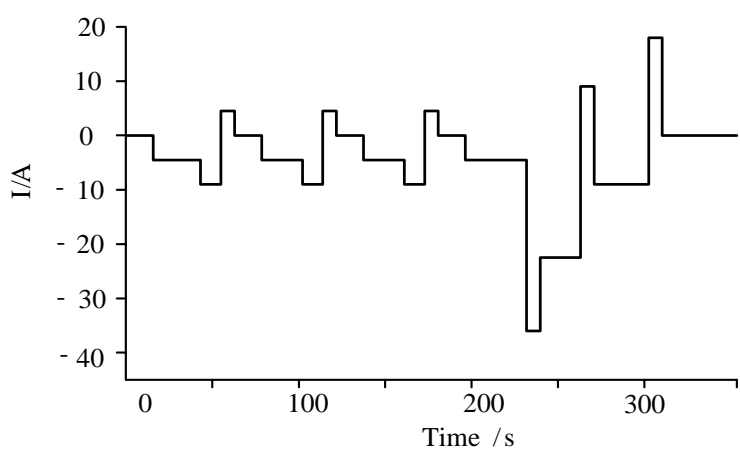

(a)

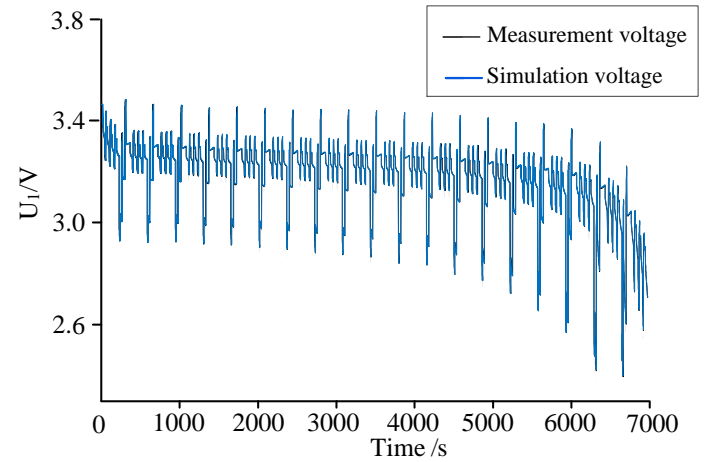

(b)

Figure 10. Model Verification under DST (dynamic stress test). (a) Current curve at DST. (b) The measurement voltage and simulation voltage.

This paper uses the mean absolute error to evaluate the fit of the battery model. The mean absolute error defined in this paper is shown as (32). The mean absolute errors are listed in Table 6.

$$
\mathrm{MAE}=\frac{1}{n} \sum_{k=1}^{n}\left|e_{k}\right|
$$

where MAE represents the mean absolute error, and $e_{k}$ represents the absolute error at time $\mathrm{k}$. It can be seen that the battery model established by the HPPC experimental method still has the advantages of high precision, small error by the analysis of the terminal voltage change of the lithium battery in the DST condition experiments in Figure 10, and mean absolute error of terminal voltage in Table 6, which fully verify the accuracy of the battery model.

Table 6. Mean absolute errors (MAE) of terminal voltage (V).

\begin{tabular}{cccccc}
\hline Temperature $\left({ }^{\circ} \mathbf{C}\right)$ & $\mathbf{- 1 0}$ & $\mathbf{0}$ & $\mathbf{1 0}$ & $\mathbf{2 5}$ & $\mathbf{4 5}$ \\
\hline MAE & 0.046 & 0.034 & 0.023 & 0.012 & 0.019 \\
\hline
\end{tabular}

\subsection{Validation of SOC Estimation Accuracy}

In order to verify the accuracy of the proposed UPF-based SOC estimation, a single $\mathrm{LiFePO}_{4}$ battery was selected, where the rated capacity is $9 \mathrm{Ah}$, and its $3 \mathrm{~A}$ current pulse discharge waveform 
is shown in Figure 11. In order to compare our UPF and existing EKF-, UKF-, and PF-based SOC estimation methods in terms of the estimation error, robustness, and other aspects by MATLAB simulations, we set the number of particles to 80 in the simulation.

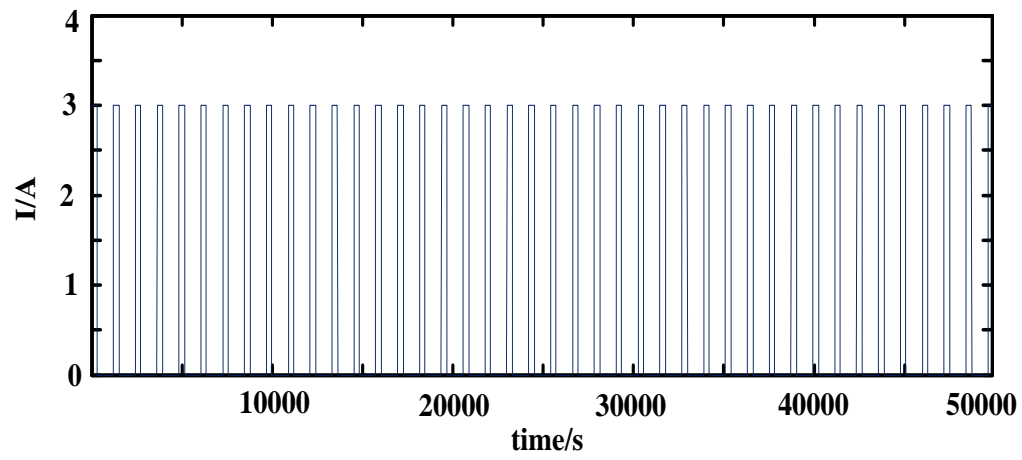

Figure 11. Discharge current curve.

Through analyzing Figures 12 and 13, it is found that the newly proposed UPF-based SOC estimation method has higher estimation accuracy and strong robustness against noises. The maximum estimation errors at $25^{\circ} \mathrm{C}$ of the estimation methods are compared in Table 7.

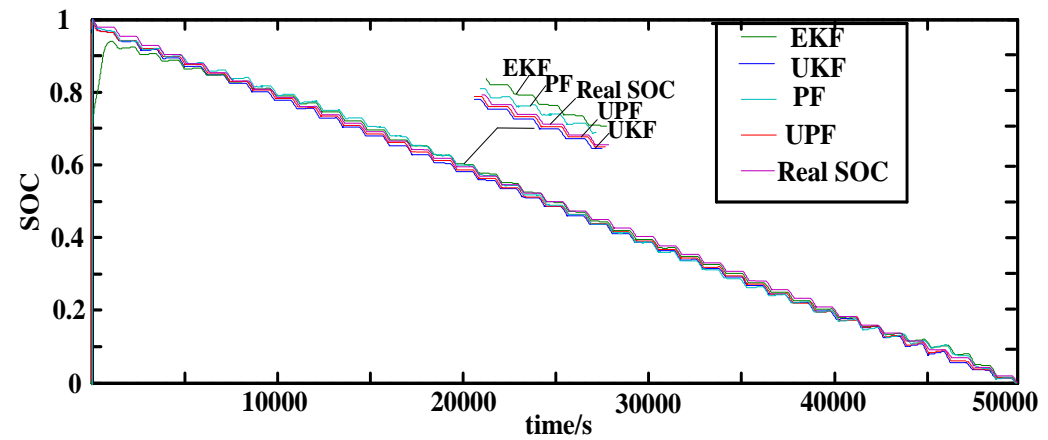

Figure 12. SOC Estimation curves of different algorithms at $25^{\circ} \mathrm{C}$. EKF, extended Kalman filter; UKF, unscented Kalman filter; PF, particle filter, UPF, unscented Kalman particle filter.

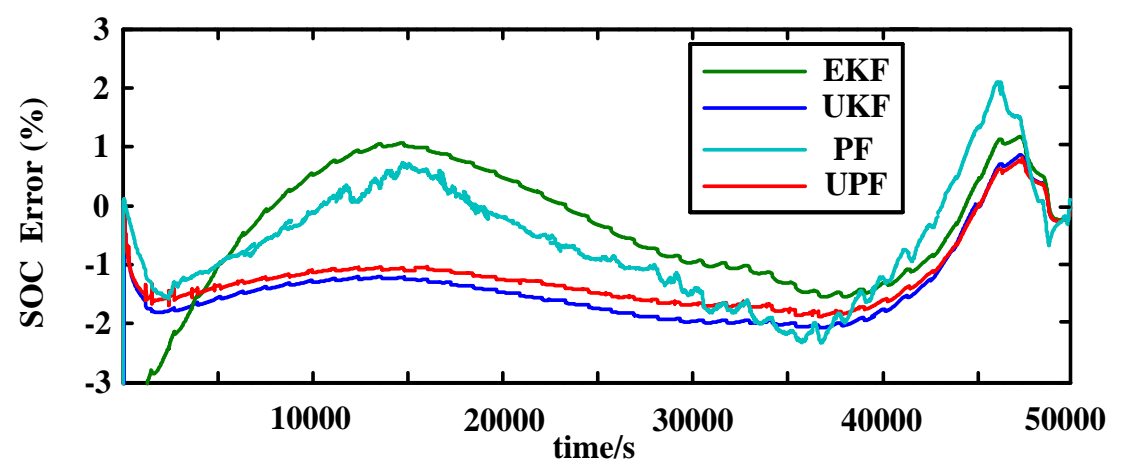

Figure 13. Estimation error curves of different algorithms at $25^{\circ} \mathrm{C}$.

Table 7. Maximum Estimation Error of Different Algorithms at $25^{\circ} \mathrm{C}$.

\begin{tabular}{ccccc}
\hline Algorithm & EKF & UKF & PF & UPF \\
\hline Maximum estimation error & $3.97 \%$ & $2.28 \%$ & $2.61 \%$ & $1.59 \%$ \\
\hline
\end{tabular}

In order to verify whether the newly proposed UPF-based SOC estimation method still has higher estimation accuracy under low temperature, a comparison of different SOC estimation methods at $-10^{\circ} \mathrm{C}$ is provided in Figure 14. 


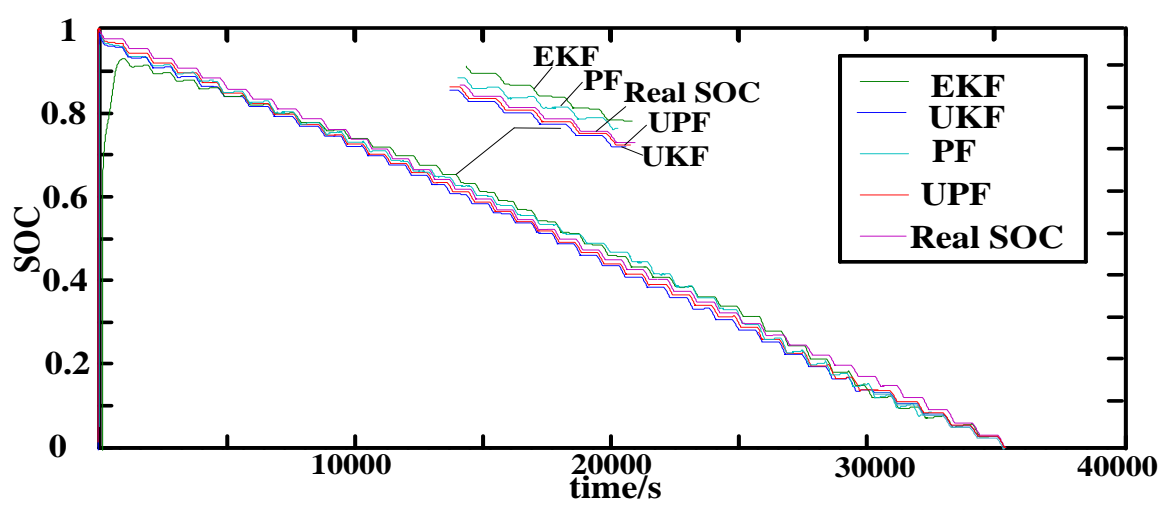

Figure 14. SOC estimation of different algorithms at $-10^{\circ} \mathrm{C}$.

Based on Figure 15 and the maximum estimation error of each estimation algorithm in Table 8, it can be found that the newly proposed UPF-based SOC estimation method still has the best accuracy at low temperature.

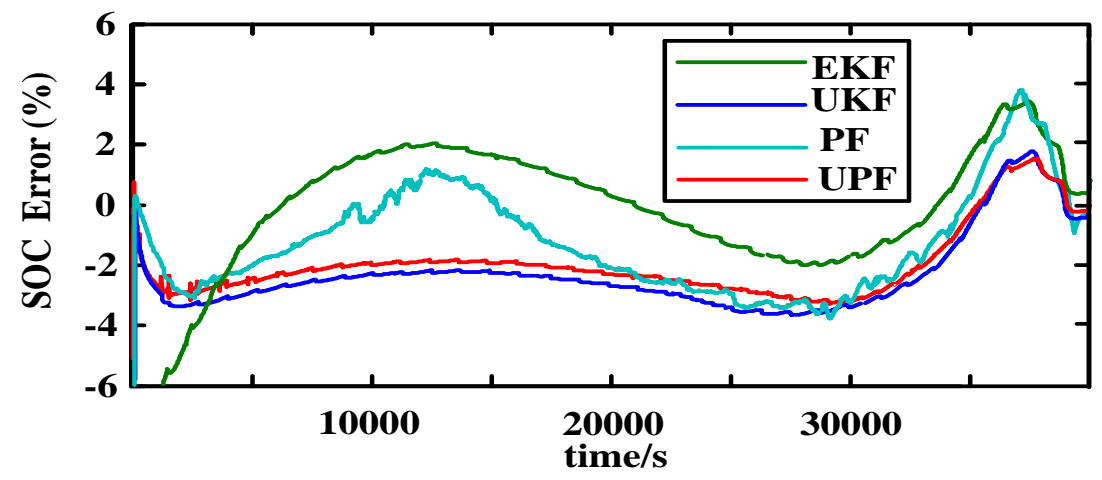

Figure 15. Estimation error curves of different algorithms at $-10{ }^{\circ} \mathrm{C}$.

Table 8. Maximum Estimation Error of Different Algorithms at $-10{ }^{\circ} \mathrm{C}$.

\begin{tabular}{ccccc}
\hline Algorithm & EKF & UKF & PF & UPF \\
\hline Maximum estimation error & $5.69 \%$ & $3.53 \%$ & $3.86 \%$ & $2.72 \%$ \\
\hline
\end{tabular}

In order to verify the robustness of the established UPF algorithm when the model parameters are under disturbances, the open-circuit voltage $U_{o c}$ was disturbed under the condition of $3 \mathrm{~A}$ pulsed current discharge in Figure 11, and the SOC estimation error curve of each algorithm was obtained, as shown in Figure 16.

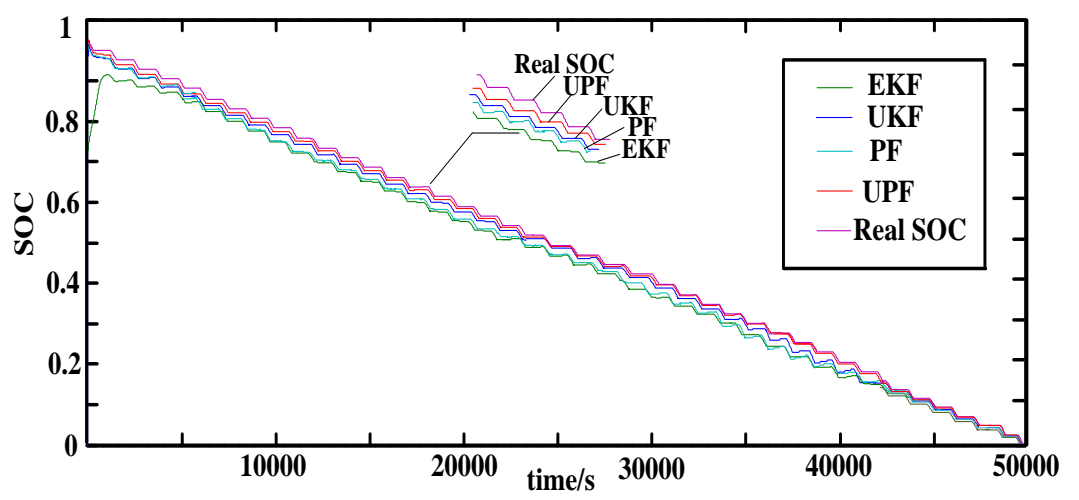

Figure 16. SOC estimation curves of different algorithms under open-circuit voltage disturbances. 
Based on Figure 17 and the maximum estimation error of each estimation algorithm in Table 9, it can be found that the newly proposed UPF-based SOC estimation method is more robust to interference caused by model parameter disturbances.

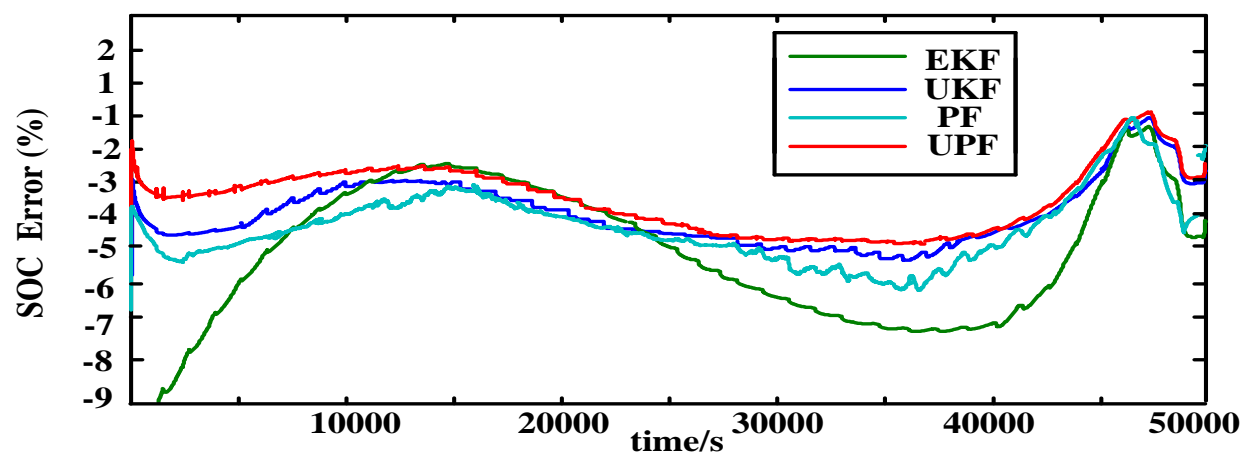

Figure 17. SOC estimation error curves of different algorithms under open-circuit voltage disturbances.

Table 9. Maximum Estimation Error of Different Algorithms under open-circuit voltage disturbances.

\begin{tabular}{ccccc}
\hline Algorithm & EKF & UKF & PF & UPF \\
\hline Maximum estimation error & $7.28 \%$ & $4.97 \%$ & $5.51 \%$ & $4.13 \%$ \\
\hline
\end{tabular}

The above verification is based on the normal charging and discharging conditions in the laboratory, however, in the actual driving process, the working conditions are random. In order to verify the effectiveness of the proposed method in the dynamic process, the comparison verification based on the dynamic conditions was added. The federal urban driving schedule (FUDS) [22] was selected to verify the proposed method. The current and voltage profiles are shown in Figure 18.

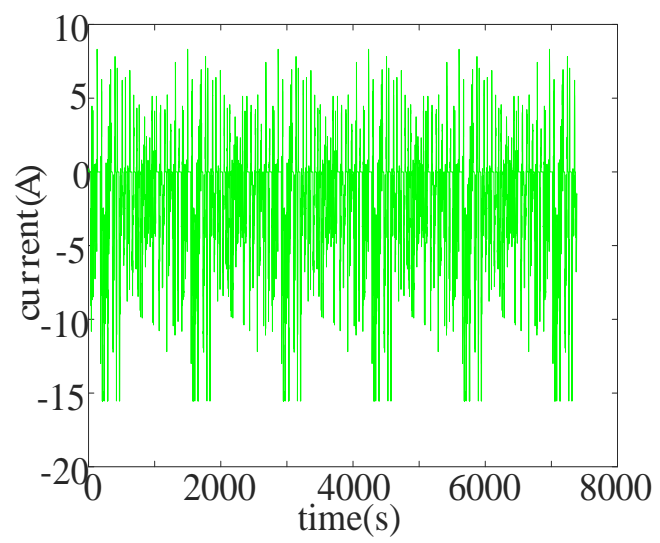

(a)

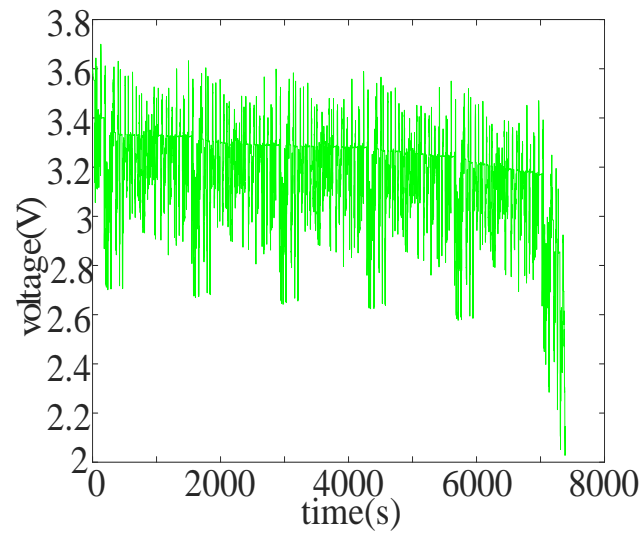

(b)

Figure 18. (a) Current curve at FUDS (federal urban driving schedule); (b) The measurement voltage.

The FUDS test was run at $25^{\circ} \mathrm{C}$. Figures 19 and 20 represent the SOC and error curves estimated by the proposed and other methods. The maximum estimation errors at $25{ }^{\circ} \mathrm{C}$ of the estimation methods are compared in Table 10. It can be seen from the analysis that the proposed method has higher accuracy than other methods in dynamic operation conditions.

Table 10. Maximum Estimation Error of Different Methods at $25^{\circ} \mathrm{C}$.

\begin{tabular}{ccccc}
\hline Algorithm & EKF & UKF & PF & UPF \\
\hline Maximum estimation error & $5.21 \%$ & $3.62 \%$ & $3.89 \%$ & $1.97 \%$ \\
\hline
\end{tabular}




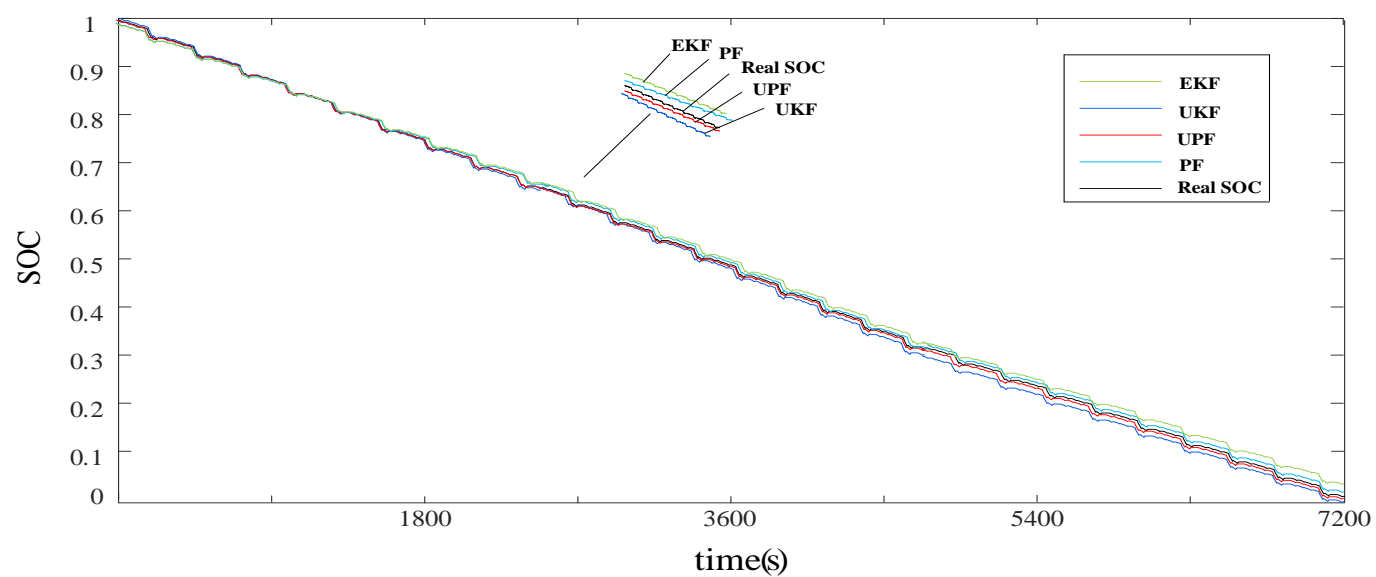

Figure 19. SOC Estimation curves of different methods.

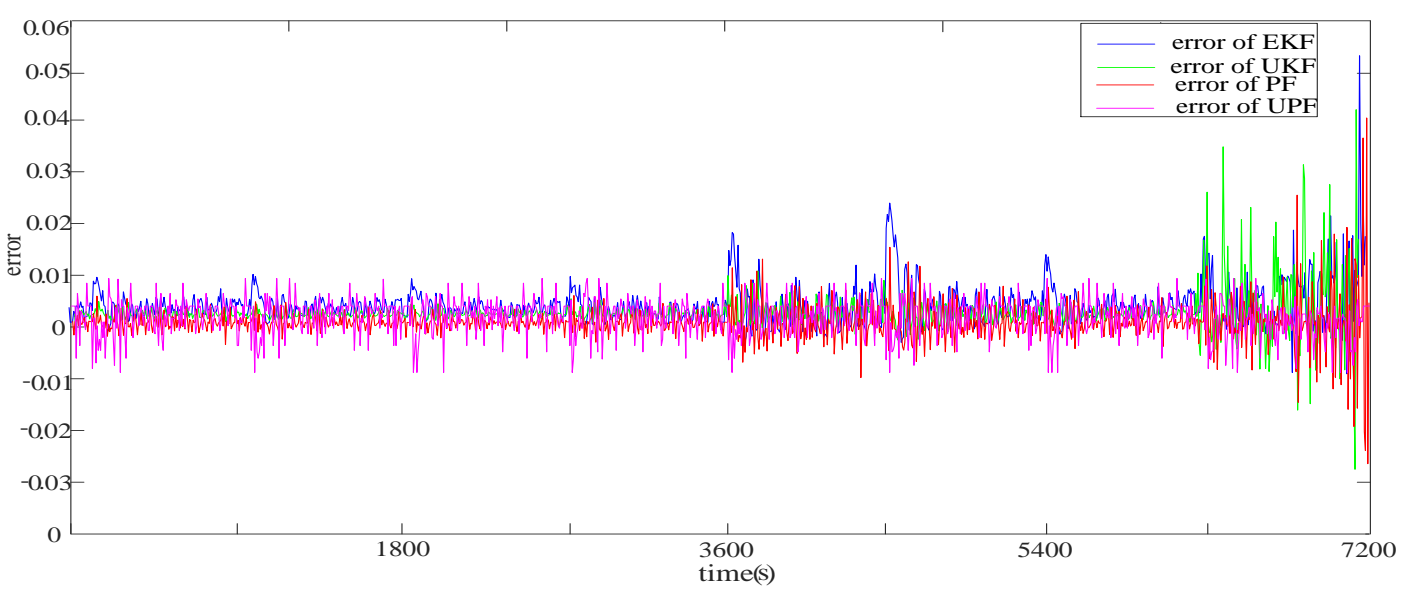

Figure 20. Estimation error curves of different methods.

To verify the accuracy of the proposed method at low temperature, the FUDS test was run at $-10{ }^{\circ} \mathrm{C}$. The results are shown in Figures 21 and 22 and Table 11.

Table 11. Maximum Estimation Error of Different Methods at $-10^{\circ} \mathrm{C}$.

\begin{tabular}{ccccc}
\hline Algorithm & EKF & UKF & PF & UPF \\
\hline Maximum estimation error & $6.73 \%$ & $4.82 \%$ & $5.18 \%$ & $3.23 \%$ \\
\hline
\end{tabular}

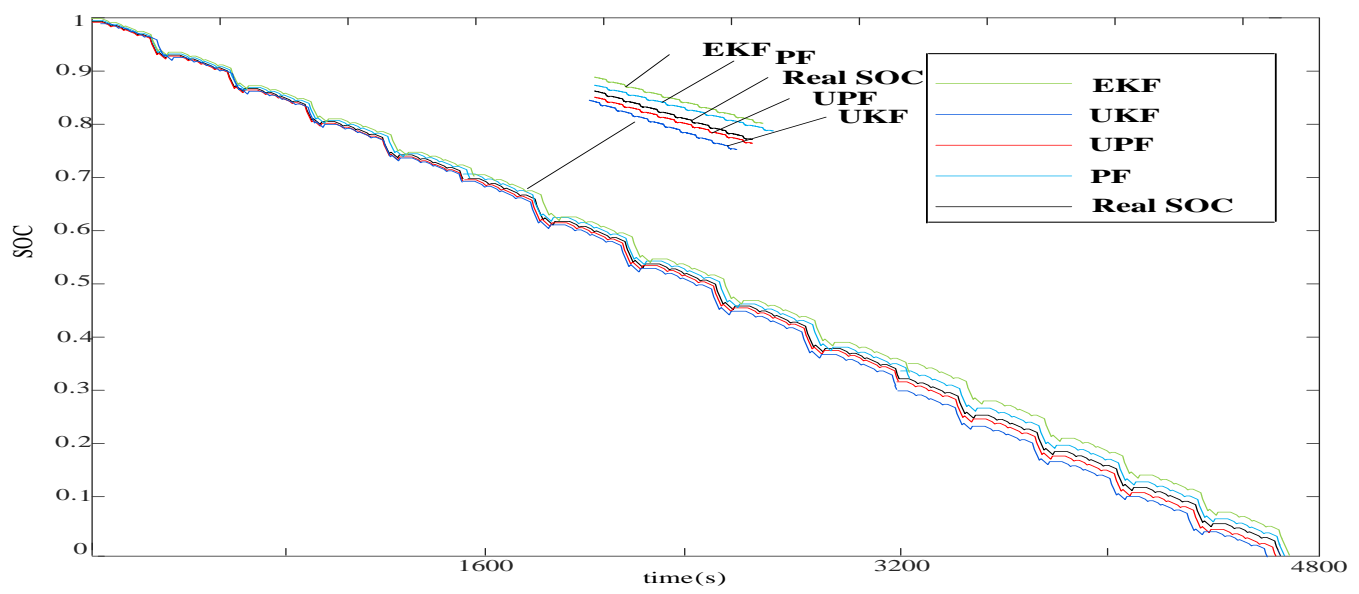

Figure 21. SOC Estimation curves of different methods. 


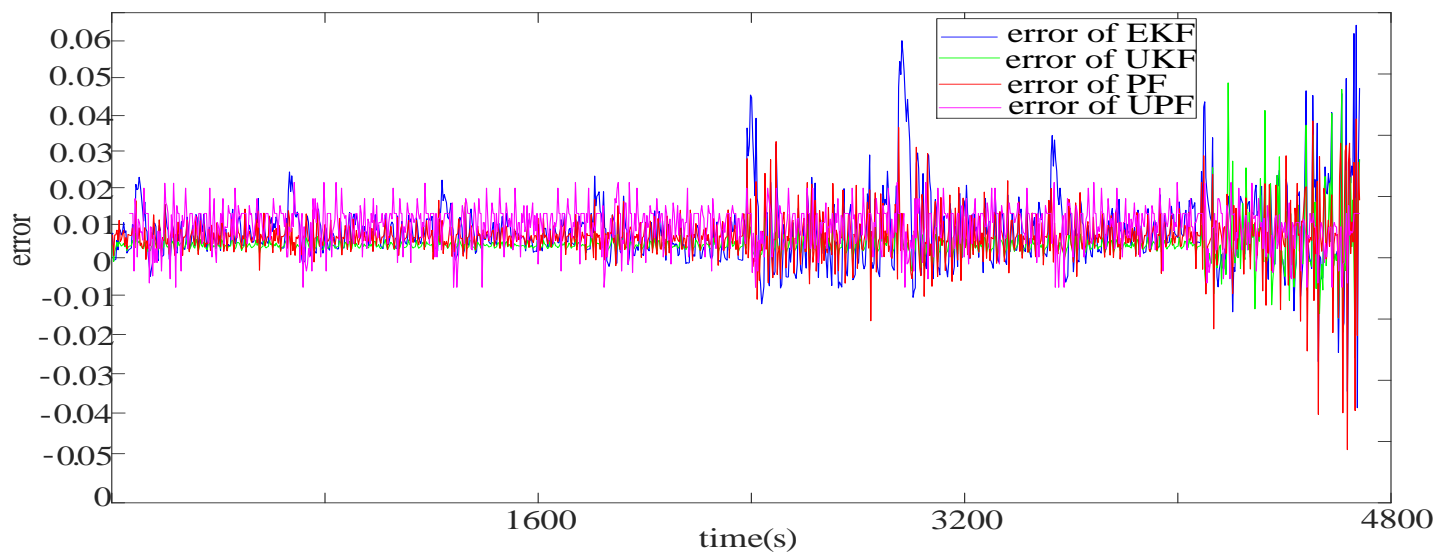

Figure 22. Estimation error curves of different methods.

From the above results, it can be seen that the proposed method still has high accuracy under the condition of low-temperature operation. According to the above analysis, the proposed method still has higher estimation accuracy than other methods under dynamic conditions.

By analyzing the estimation curves and error curves of each algorithm in each state in the above simulation experiment, it can be found that the UPF-based SOC estimation has higher estimation accuracy than other commonly used methods, and it also has better anti-interference performance for the disturbance caused by model parameters.

\section{Conclusions}

In order to obtain a SOC estimation method with high accuracy and wide temperature application range, the UPF algorithm and the variable parameter battery Thevenin model are adopted in this paper to propose a new SOC estimation method. This new method has the following features.

The parameters of the battery model are identified by using the parameters fitting method of CCD-based DOE and least squares method to achieve $95 \%$ confidence level according to the variations of the internal resistance. The obtained variable-parameter Thevenin model can also accurately characterize the influence of temperature, $T$, and SOC, thus ensure the accuracy of the battery model.

The parameter fitting method used here can obtain a more accurate battery model, based on less measured data, and reduce the workload while ensuring the accuracy of the battery model. Compared with other SOC estimation methods, the UPF-based SOC estimation method can estimate battery SOC in real time and has stronger anti-interference performance.

Author Contributions: Conceptualization: X.L.; methodology: X.D.; software: Y.H., X.Z.; validation, G.Z. All authors have read and agreed to the published version of the manuscript.

Funding: This research was funded by the National Natural Science Foundation, grant number [61603120].

Conflicts of Interest: The authors declare no conflict of interest.

\section{References}

1. Chaoui, H.; Gualous, H. Adaptive state of charge estimation of lithium-ion batteries with parameter and thermal uncertainties. IEEE Trans. Control Syst. Technol. 2017, 25, 752-759. [CrossRef]

2. Yang, R.; Xiong, R.; He, H.W.; Mu, H.; Wang, C. A novel method on estimating the degradation and state of charge of lithium-ion batteries used for electrical vehicles. Appl. Energy 2017, 207, 331-340. [CrossRef]

3. Pan, R.; Wang, Y.J.; Zhang, X.; Yang, D.; Chen, Z.H. Power capability prediction for lithium-ion batteries based on multiple constraints analysis. Electrochim. Acta 2017, 238, 120-133. [CrossRef]

4. He, Y.; Liu, X.T.; Zhang, C.B.; Chen, Z.H. A new model for State-of-Charge estimation for high-power Li-ion batteries. Appl. Energy 2013, 101, 808-814. [CrossRef]

5. Zou, C.; Hu, X.; Wei, Z.; Wik, T.; Egardt, B. Electrochemical estimation and control for lithium-ion battery health-aware fast charging. IEEE Trans. Ind. Electron. 2018, 65, 6635-6645. [CrossRef] 
6. Xiong, R.; Cao, J.; Yu, Q.Q.; He, H.W.; Sun, F.C. Critical review on the battery State of Charge estimation methods for electric vehicles. IEEE Access 2018, 6, 1832-1842. [CrossRef]

7. Xing, Y.J.; He, W.; Pecht, M.; Tsui, K.L. State of charge estimation of lithium-ion batteries using the open-circuit voltage at various ambient temperatures. Appl. Energy 2014, 113, 106-115. [CrossRef]

8. Kang, L.; Zhao, X.; Ma, J. A new neural network model for the state-of-charge estimation in the battery degradation process. Appl. Energy 2014, 121, 20-27. [CrossRef]

9. Liu, X.T.; He, Y.; Zheng, X.X.; Zhang, J.F.; Zeng, G.J. A new State-of-Charge estimation method for electric vehicle Lithium-Ion batteries based on multiple input parameter fitting model. Int. J. Energy Res. 2017, 41, 1265-1276. [CrossRef]

10. Sun, F.C.; Xiong, R.; He, H.W. Estimation of state-of-charge and state-of-power capability of lithium-ion battery considering varying health conditions. J. Power Sources 2014, 259, 166-176. [CrossRef]

11. Xu, J.; Mi, C.; Cao, B.G.; Cao, J.Y. A new method to estimate the state of charge of lithium-ion batteries based on the battery impedance model. J. Power Sources 2013, 233, 277-284. [CrossRef]

12. Plett, G. Extended Kalman filtering for battery management systems of LiPB-based HEV battery packs: Part 3. State and parameter estimation. J. Power Sources 2004, 134, 277-292. [CrossRef]

13. He, Z.W.; Cao, M.Y.; Wang, C.S.; Wang, L.Y.; Liu, Y.Y. Adaptive state of charge estimation for Li-ion batteries based on an unscented Kalman filter with an enhanced battery model. Energies 2013, 6, 4134-4151. [CrossRef]

14. Wang, Y.J.; Zhang, C.B.; Chen, Z.H. A method for state-of-charge estimation of $\mathrm{LiFePO}_{4}$ batteries at dynamic currents and temperatures using particle filter. J. Power Sources 2015, 279, 306-311. [CrossRef]

15. Xiong, R.; He, H.W.; Zhao, K. Research on an online identification algorithm for a Thevenin battery model by an experimental approach. Int. J. Green Energy 2015, 12, 272-278. [CrossRef]

16. Chin, C.S.; Gao, Z.; Chiew, J.H.K.; Zhang, C. Nonlinear temperature-dependent state model of cylindrical $\mathrm{LiFePO}_{4}$ battery for open-circuit voltage, terminal voltage and state-of-charge estimation with extended Kalman filter. Energies 2018, 11, 2467. [CrossRef]

17. Zheng, X.X.; Xiao, L.; Lei, Y.; Wang, Z.L. Optimization of LCL filter based on closed-loop total harmonic distortion calculation model of the grid-connected inverter. IET Power Electron. 2015, 27, 87-94.

18. Wang, W.Z.; Malysz, P.; Khan, K.; Gauchia, L.; Emadi, A. Modeling, parameterization, and benchmarking of a lithium-ion electric bicycle battery. In Proceedings of the IEEE Energy Conversion Conversion Congress and Exposition (ECCE), Milwaukee, WI, USA, 18-22 September 2016.

19. Feng, F.; Lu, R.; Wei, G.; Zhu, C. Online estimation of model parameters and state of charge of $\mathrm{LiFePO}_{4}$ batteries using a novel open-circuit voltage at various ambient temperatures. Energies 2015, 8, 2950-2976. [CrossRef]

20. Li, Y.; Wang, C.; Gong, J. A combination Kalman filter approach for the state of charge estimation of lithium-ion battery considering model uncertainly. Energies 2016, 109, 933-946. [CrossRef]

21. Liu, X.T.; Chen, Z.H.; Zhang, C.B.; Wu, J. A novel temperature-compensated model for power Li-ion batteries with dual-particle-filter state-of-charge estimation. Appl. Energy 2014, 123, 263-272. [CrossRef]

22. He, W.; Williard, N.; Chen, C.C.; Pecht, M. State of charge estimation of lithium-ion batteries using neural network modeling and unscented Kalman filter-based error cancellation. Int. J. Electr. Power Energy Syst. 2014, 62, 783-791. [CrossRef]

(C) 2019 by the authors. Licensee MDPI, Basel, Switzerland. This article is an open access article distributed under the terms and conditions of the Creative Commons Attribution (CC BY) license (http://creativecommons.org/licenses/by/4.0/). 